\title{
The Bad Use of Metaphors and the Use of Bad Metaphors
}

\author{
Kostas Kampourakis ${ }^{1}$
}

Published online: 9 January 2017

(C) Springer Science+Business Media Dordrecht 2017

Scientists commonly use metaphors in order to describe and explain natural phenomena, processes, or mechanisms. For example, physicists talk about "waves" although there is no medium in which they move and "particles" even though these are not really solid. Metaphors make these more comprehensible for non-experts because they are guided to think about them in terms of other phenomena, processes, or mechanisms they are familiar with. However, a major problem that emerges for science education and communication is that it is often very easy to confuse the metaphor with the actual phenomenon. Worse than that, the focus may eventually be on those elements of the actual phenomenon in which the metaphor is better illustrated. As a result, people may overlook other, perhaps important, aspects that do not fit well in the metaphor.

One domain in which metaphors have been useful is genetics research. This has certainly been the case for the metaphor of "information" encoded in DNA that paved the way for deciphering the genetic code, or the metaphor of the genome as the "book of life" on which the human genome project was based. Several other metaphors have been employed: DNA analysis has been described as "reading"; DNA replication has been described as "copying"; RNA synthesis has been described as "transcription," protein synthesis has been described as "translation," RNA modification has been described as "editing," and more. Such metaphors are not inherently wrong and can actually help us make sense of the respective phenomena. But especially non-experts should always keep in mind that metaphors are a means of representation and nothing more. "Books," "software," "reading," "writing," etc. are all human inventions and thus have an inherent component of anthropomorphism. This needs to be made explicit or we should otherwise avoid any unnecessary use of expressions of this kind.

If this does not happen, metaphors can mislead. Genes are an exemplar case of the negative impact that the bad use of metaphors and the use of bad metaphors can have. Many of the metaphors currently used about genes present them as autonomous entities, which both contain all the necessary information to determine characters and can make use of it. Two metaphors have been mainly used by biologists and non-biologists in order to account for what genes

Kostas Kampourakis

Kostas.Kampourakis@unige.ch 
"do": the genome has been described as a book and as a genetic program. For instance, the announcement of the first sequence of the human genome in 2000 as the outcome of the Human Genome Project was presented in BBC under the title "Reading the book of life," actually using both of these metaphors in the first lines: "The blueprint of humanity, the book of life, the software for existence - whatever you call it, decoding the entire three billion letters of human DNA is a monumental achievement." ${ }^{1}$ The genome was thus perceived both as a book containing information and as a program through which this information is used. In a similar manner, the ENCODE (Encyclopedia of DNA Elements) project was presented in 2012 in CNN under the title "DNA project interprets 'book of life'," in which it was stated that: "When the Human Genome Project sequenced the human genome in 2003, it established the order of the 3 billion letters in the genome, which can be thought of as 'the book of life'."2 It must be noted at this point that this metaphor is inherent in the notion of the "encyclopedia" employed by the researchers of the ENCODE project themselves.

This kind of thinking about genes has often made experts forget how much we still do not know about genes and non-experts to think of genes as the "essences" of life, as the absolute "determinants" of characters and disease and therefore as providing the ultimate explanations for all biological phenomena because the latter can be "reduced" to the gene level and thus be explained. It is therefore necessary for researchers and educators to always be explicit about the limits of the metaphors they use. We can say that genes "encode" some "functional" products, insofar as we clearly explain that this is just a way of representing the informational properties of DNA, which are not inherent and which make sense only in the cellular context in which they can be used as a resource for the production of molecules. We should also explain that often metaphors are used because we ignore the details and so they have a heuristic value both in explaining the respective phenomena and in guiding further research. Therefore, what we need to do is to use appropriate metaphors as well as always be explicit about their nature and limitations. For example, if we describe genes as DNA sequences encoding information for functional products, be it proteins or RNA molecules, we should be explicit that with "encoding information," we mean that the DNA sequence is used as the template to produce an RNA molecule or a protein that performs some function. We should also be explicit that "encoding information" is not an inherent property of DNA, but one that exists only in its (intra- and inter-) cellular context. Even if one preferred to generally refrain from using anthropomorphic metaphors, "encoding information" has been a widely used expression that really makes sense to most people. It is not possible to entirely refrain from using metaphors, so we should be explicit about them and appropriately use them in well-defined frameworks, rather than inappropriately building the frameworks on those metaphors.

Therefore, scientists need to be aware of how non-experts think about genes: to use appropriate metaphors and to be explicit about their limitations, and to actively participate in education and outreach activities. These in turn require a broader culture and studies than what undergraduate studies usually provide. Concepts and conceptual issues should be given more attention in education and outreach. This would be the first step for an effective "translation" of these concepts from the scientific context to the public sphere. Metaphors will always be there, but we can make an appropriate use of good metaphors that will help non-experts make sense of scientific concepts, and more broadly of scientific research. This is a much-needed step before anyone attempts to understand the impact of this research for one's life.

\footnotetext{
${ }^{1}$ http://news.bbc.co.uk/2/hi/indepth/scitech/2000/humangenome/760893.stm

${ }^{2}$ http://edition.cnn.com/2012/09/05/health/encode-human-genome/
} 
It is at this point that science educators have a very crucial contribution to make. Especially those of us who work in science education and tend to draw on history, philosophy, and sociology of science, might contribute to bridging the two worlds: make researchers aware of how non-experts think and of the important conceptual issues at stake and also appropriately present scientific research in ways that non-experts understand it without misleading or altering the actual knowledge. I envision science educators writing several books that would help non-experts make sense of several important scientific concepts such as force, energy, adaptation, and more. For a start, Science \& Education would welcome articles on the bad use of metaphors and the use of bad metaphors in science education and communication.

\section{Compliance with ethical standards}

Conflict of interest The author declares no conflict of interest. 